\title{
X-ray photon correlation spectroscopy revealing the change of relaxation dynamics of a severely deformed Pd-based bulk metallic
}

\author{
glass \\ Hongbo Zhou ${ }^{\mathrm{a}^{*}}$, Sven Hilke ${ }^{\mathrm{a}}$, Eloi Pineda ${ }^{\mathrm{b}}$, Martin Peterlechner ${ }^{\mathrm{a}}$, Yuriy Chushkin ${ }^{\mathrm{c}}$, \\ Sankaran Shanmugam ${ }^{\mathrm{d}}$ and Gerhard Wilde $\mathrm{a}^{\mathrm{a}^{* *}}$ \\ anstitute of Materials Physics, University of Muenster, Wilhelm-Klemm Strasse 10, \\ Muenster 48149, Germany \\ bepartment of Physics, Institute of Energy Technologies, Universitat Politècnica de \\ Catalunya - BarcelonaTech, Av. Eduard Maristany 16, Barcelona 08019, Spain \\ 'European Synchrotron Radiation Facility, Avenue des Martyrs 71, Grenoble 38000, \\ France \\ ${ }^{\mathrm{d} D e p a r t m e n t ~ o f ~ M e t a l l u r g i c a l ~ a n d ~ M a t e r i a l s ~ E n g i n e e r i n g, ~ I I T ~ M a d r a s, ~ C h e n n a i ~ 600036, ~}$ \\ India
}

To whom correspondence should be addressed. E-mail:

*h_zhou04@uni-muenster.de; ${ }^{* *}$ gwilde@uni-muenster.de

\begin{abstract}
The influence of severe plastic deformation on the relaxation dynamics of a $\mathrm{Pd}_{40} \mathrm{Ni}_{40} \mathrm{P}_{20}$ (at.\%) bulk metallic glass was investigated on the atomic length scale by X-ray photon correlation spectroscopy and fluctuation electron microscopy. At a series of isothermal temperatures adjusted by step heating below the glass transition, the relaxation times were obtained by X-ray photon correlation spectroscopy and the
\end{abstract}


corresponding activation energies were evaluated. It was found that the relaxation dynamics was accelerated by about a half order of magnitude after plastic deformation by high-pressure torsion processing, which indicates that effective rejuvenation has occurred. At relatively low temperatures the relaxation process was stress-dominated and deformation could improve the atomic mobility without changing the energy barrier. At higher temperatures, a second regime of relaxation times occurred. It is concluded that with increasing temperature, a crossover from a stress-dominated to a diffusion-dominated relaxation process occurs. Moreover, the medium range order was measured using variable resolution fluctuation electron microscopy indicating that deformation-induced changes in topology are responsible for the rejuvenation and the accelerated dynamics.

Keywords: Bulk metallic glass; High-pressure torsion; X-ray photon correlation spectroscopy; Relaxation dynamics; Fluctuation electron microscopy; Medium range order

\section{Introduction}

The study of structural relaxation processes in metallic glasses (MGs) is a subject of continuous scientific and technological interest since the relaxation behavior of glasses presents the key for fine tuning properties in a manner which is inaccessible for crystalline materials. The practical significance of the subject is also evident from the close connection between the relaxation behavior and the mechanical properties such as 
embrittlement and catastrophic mechanical failure [1, 2]. Numerous investigations have been undertaken with the purpose of characterizing the relaxation in MGs and elucidating the structural origin underlying it [3, 4]. Even though in the past decades there have been plenty of efforts and developments towards the understanding of the nature of glass relaxation [4-9], a tangible picture based on an atomic-level description has not yet been obtained and there exist sometimes apparently contradictory interpretations of the same processes [10-13].

The structural rearrangements involved in relaxation are directly related to the variation of density fluctuations in the system, which may be described by the density correlation function [14]. Therefore, characterizing the density correlation function and its dependence on time and temperature is a fundamental approach to probe the dynamics of structural rearrangements on the atomic length scale during the relaxation process. Quasi-elastic neutron scattering and Mößbauer spectroscopy have been used to study the atomic dynamics [15], but these methods suffer from the limitation of available isotopes and unsuitability for the slow dynamics below the glass transition temperature $\left(\mathrm{T}_{\mathrm{g}}\right)$. Thus, there is a need for a universal method to study the relaxation dynamics, which is also capable to track slower dynamics.

X-ray photon correlation spectroscopy (XPCS) is a novel technique which is highly sensitive to the aforementioned issue and has been used in recent years to probe the microscopic dynamics of MGs [14, 16-18]. XPCS records the temporal evolution of the 
diffracted intensity when a coherent X-ray beam passes through the sample foil and the observed intensity fluctuations derive directly from the atomic rearrangements in MGs. This method has been applied to study some intriguing phenomena, such as aging behavior, intermittent relaxation in hyper-quenched MGs and the anti-aging behavior in ultrastable MGs [14, 18, 19]. As shown, plastic deformation induces atomic rearrangements and influences the thermodynamics of glasses and polymers [20-24], however in a yet unknown way. Therefore, in this study we report the influence of severe plastic deformation on the relaxation behavior and the atomic structure of MGs using XPCS.

The novelty of this work is that XPCS measurements were carried out systematically at a series of isothermal temperatures during step heating from $290 \mathrm{~K}$ to compare the relaxation dynamics of an already well-relaxed MG and a highly deformed MG by high-pressure torsion (HPT). In addition, variable resolution fluctuation electron microscopy (VR-FEM) was performed to characterize the HPT-induced variations of medium-range order (MRO) locally. Information on the MRO sizes and relative volume fractions was extracted from a statistically relevant number of nano-beam diffraction patterns (NBDPs) using parallel coherent probe sizes[25-29]. The unique combination of VR-FEM and time-resolved XPCS techniques gives an opportunity for elucidating the atomic rearrangements during HPT and the influence of deformation on the subsequent relaxation dynamics on the relevant -, i.e. the atomic length scale. 


\section{Experimental}

\subsection{Sample preparation}

Palladium-based MGs have an outstanding glass-forming ability and relatively good plasticity, and thus are particularly suitable for studying deformation and post-deformation properties of MGs [30-34]. To prepare $\mathrm{Pd}_{40} \mathrm{Ni}_{40} \mathrm{P}_{20} \mathrm{MGs}$, palladium plates and granular $\mathrm{Ni}_{2} \mathrm{P}$ were pre-melted and homogenized in a silica crucible by induction heating under a Ti-gettered argon atmosphere, and then the material was reheated in another silica crucible with a round nozzle and injected into a copper mold to obtain a rod of $5 \mathrm{~mm}$ in diameter for HPT deformation. Prior to all experiments, the rod was annealed at $533 \mathrm{~K}$ for $6 \mathrm{~h}$ (defined as the annealed state/undeformed state). Disks with a diameter of $5 \mathrm{~mm}$ and a thickness of $0.85 \mathrm{~mm}$ cut from the annealed rod were subjected to 10, 100 and 750 revolutions using HPT under an applied hydrostatic pressure of $12 \mathrm{GPa}$ at room temperature (abbreviated as Ann_N10 and Ann_N100 and Ann_N750, respectively). Since the samples were cut from the half-radius region of the HPT discs for the XPCS and VR-FEM measurements, the equivalent shear strain was obtained by the equation $\varepsilon_{e q}=\pi r N / h$ (r: radius of the disc, $\mathrm{N}$ : number of revolutions, h: disc thickness). The equivalent strains of the Ann_10, Ann_100 and Ann_750 samples are approximately 90, 920 and 6930, respectively. It should be emphasized that this confined deformation geometry prevents catastrophic failure, enabling these outstandingly high strains [34]. All the samples were characterized by 
X-ray diffraction (XRD, Siemens D5000) confirming the X-ray amorphous nature as shown in Fig. S1 (see Supplementary Materials). XPCS samples were carefully ground to a thickness of $20 \mu \mathrm{m}$ and TEM samples were prepared by a Struers Tenupol 5 twin jet electro-polisher with the electrolyte BK-2 [35] at a voltage of $30 \mathrm{~V}$ and a temperature of $243 \mathrm{~K}$.

\subsection{X-ray photon correlation spectroscopy}

XPCS measurements were performed at the beam line ID10 at the European Synchrotron Radiation Facility (ESRF, France). The sample with a thickness of approximately $20 \mu \mathrm{m}$ was mounted in a furnace with a temperature stability better than $0.05 \mathrm{~K}$. Both the heating and cooling rates between each isothermal temperature were 6 $\mathrm{K} / \mathrm{min}$. A coherent beam with a size of $10 \times 10 \mu \mathrm{m}$ and a fixed photon energy of $8.1 \mathrm{keV}$ $(\lambda=1.53 \AA)$ illuminated the samples and the diffracted intensities were recorded in transmission after every 5 seconds by a CCD detector (Andor Ikon-M, 1024×1024 pixels, $13 \mu \mathrm{m}$ pixel size) placed $70 \mathrm{~cm}$ behind the sample position at a wave vector $\mathrm{q}_{0}=2.87 \AA^{-1}$, where the diffracted intensity manifests a maximum corresponding to the average atomic distance of the $\mathrm{Pd}_{40} \mathrm{Ni}_{40} \mathrm{P}_{20} \mathrm{MG}$.

If the sample structure changes over time, the corresponding diffraction pattern will also display a time-dependent variation. The intensity fluctuations of these patterns thus provide the information on the time-dependent dynamics of the sample. Information about the instantaneous dynamics during the isothermal process could be accessed by 
calculating the two-time correlation function (TTCF) defined by:

$$
\mathrm{g}_{2}\left(\mathrm{t}_{1}, \mathrm{t}_{2}\right)=\frac{\overline{\left\langle\mathrm{I}_{\mathrm{t} 1} \mathrm{I}_{\mathrm{t} 2}\right\rangle}}{<\overline{\mathrm{I}_{\mathrm{t} 1}}><\overline{\mathrm{I}_{\mathrm{t} 2}}>}
$$

$I_{t 1}$ and $I_{t 2}$ are the X-ray intensities collected at times $t_{1}$ and $t_{2}$ at the wave vector $q_{0}$ and the brackets $<\ldots>$ denote the ensemble average over all the pixels of the detector [36]. In the TTCF representation, the diagonal direction indicates the evolution of the measurement time and the distance from the diagonal is proportional to the lag time $t=$ $\left|t_{1}-t_{2}\right|$. The quantitative evaluation of the dynamics is achieved by calculating the time averaged autocorrelation function expressed as [37, 38]:

$$
g_{2}(t)=1+B\left(q_{0}\right)\left[f\left(q_{0}, t\right)\right]^{2}
$$

based on the extension of Siegert's relation to non-ergodic systems [37, 38]. B(q) is a setup-dependent factor [39]. Eq. (2) could also be fitted by a Kohlrausch-Williams-Watts (KWW) function:

$$
f(t)=1+A \cdot \exp \left[-2(t / \tau)^{\beta}\right]
$$

$\tau$ and $\beta$ in Eq. (3) denote the relaxation time and stretching exponent, respectively.

2.3. Variable resolution fluctuation electron microscopy

VR-FEM experiments were conducted in a transmission electron microscope (TEM, Thermo Fisher FEI Themis G3 60-300) operated at an acceleration voltage of $300 \mathrm{kV}$. NBDPs were acquired with different probe sizes $(0.8-4.2 \mathrm{~nm})$ at full width half maximum (FWHM). The probe size was varied via the semi-convergence angle [40]. More details for the experimental setup of VR-FEM are given in [27]. 
Electron energy loss spectroscopy (EELS) was used to calculate the thickness of the investigated areas by applying the log-ratio method [41] in order to keep experimental parameters for investigations equal, i.e. thickness, beam current and exposure time. The spatially resolved diffraction intensity $\mathrm{I}(\vec{k}, \mathrm{R}, \vec{r})$ of the acquired NBDPs was analyzed statistically, where $\vec{k}$ is the diffraction vector, $\mathrm{R}$ is the probe size, and $\vec{r}$ is the individual position on the sample. The analysis is calculated on a pixel by pixel basis [42]. Subsequently, the normalized variance profiles $\mathrm{V}(\vec{k}, \mathrm{R})$ were calculated using the following formula:

$$
\mathrm{V}(\vec{k}, \mathrm{R})=\frac{<\mathrm{I}^{2}(\vec{k}, \mathrm{R}, \vec{r})>}{<\mathrm{I}(\vec{k}, \mathrm{R}, \vec{r})>^{2}}-1
$$

where $\left\langle\mathrm{I}^{2}(\vec{k}, \mathrm{R}, \vec{r})\right\rangle$ is the mean square intensity and $\langle\mathrm{I}(\vec{k}, \mathrm{R}, \vec{r})>$ denotes the averaged intensity over all NBDPs [28].

The obtained results from VR-FEM were analyzed in the framework of the para-crystalline model, which idealizes the amorphous state as an undercooled liquid composed of small para-crystallites as sub-structural units or motifs embedded in liquid-like matter. MRO is then defined, when such sub-structural units or motifs are correlated beyond the next-nearest neighbor distance. In this work the MRO was analyzed in detail using the Stratton-Voyles approach (see Eq. 5) which assumes the variance $V(k, R)$ to follow a dependence with $1 / R^{2}[29,43]$. Plotting $V(k, R)$ versus $1 / R^{2}$ has the advantage to reveal multiple MRO correlation length by the separation of intercept- and slope-dominated parts: 


$$
\mathrm{V}(|\overrightarrow{\mathrm{k}}|, \mathrm{R}) \approx \mathrm{a}+\mathrm{b} * \frac{1}{\mathrm{R}^{2}} .
$$

\subsection{Calorimetric measurements}

Conventional differential scanning calorimetry (DSC, Perkin Elmer Diamond) was performed at a heating rate of $20 \mathrm{~K} / \mathrm{min}$ under a constant argon flow. Approximately 20 mg of the material was sampled for each DSC measurement. The glass transition temperature $T_{\mathrm{g}}$ is defined as the inflection point of the calorimetric glass transition signal during the scan. For both annealed (undeformed) and HPT deformed samples, $\mathrm{T}_{\mathrm{g}}$ amounted to $584 \mathrm{~K}$.

\section{Results}

\subsection{XPCS measurements on the annealed state}

The time-resolved microscopic dynamics related to the structural rearrangements during the relaxation can be directly monitored through the intensity fluctuations. The TTCF reflects the statistical similarity between the structural configurations measured at times $t_{1}$ and $t_{2}$, thus the discrepancy between these intensities indicates dynamics. The TTCFs of the annealed sample measured at $373 \mathrm{~K}, 423 \mathrm{~K}, 473 \mathrm{~K}$ and $523 \mathrm{~K}$ upon step heating are shown in Fig. 1. The diagonal lines from the lower left to the upper right corners represent the elapsed time during the measurements, and the width of the diagonal profile is proportional to the relaxation time $\tau$. The contrast values of the TTCF decrease as the difference between $t_{1}$ and $t_{2}$ increases, corresponding to the stepwise red-yellow-blue color change. From Fig. 1 (a), it is observed that the diagonal width is 
relatively wide and constant, which means a slow and stationary dynamics is dominating at $373 \mathrm{~K}$. The dynamics accelerates as the temperature increases to $423 \mathrm{~K}$ in Fig. 1 (b). At this temperature the dynamics are almost stationary, with slight fluctuations observed in the TTCF which may resemble intermittent dynamics found in other metallic glasses in a comparable temperature region [14]. When the temperature is raised to $473 \mathrm{~K}$, much faster dynamics is observed, accompanied by a significant aging phenomenon in Fig. 1 (c). In order to quantify the aging phenomenon at $473 \mathrm{~K}$ in more detail, normalized $\mathrm{g}_{2}(\mathrm{t})$ functions extracted from different stages of the isothermal process were evaluated, as shown in Fig. 2 (b), obtaining the relaxation times of $90 \mathrm{~s}$ for the early 2000 seconds waiting time and of 233 s for the final 2000 seconds waiting time. However, a further increase in temperature to $523 \mathrm{~K}$ leads to the deceleration of the dynamics in Fig. 1 (d).
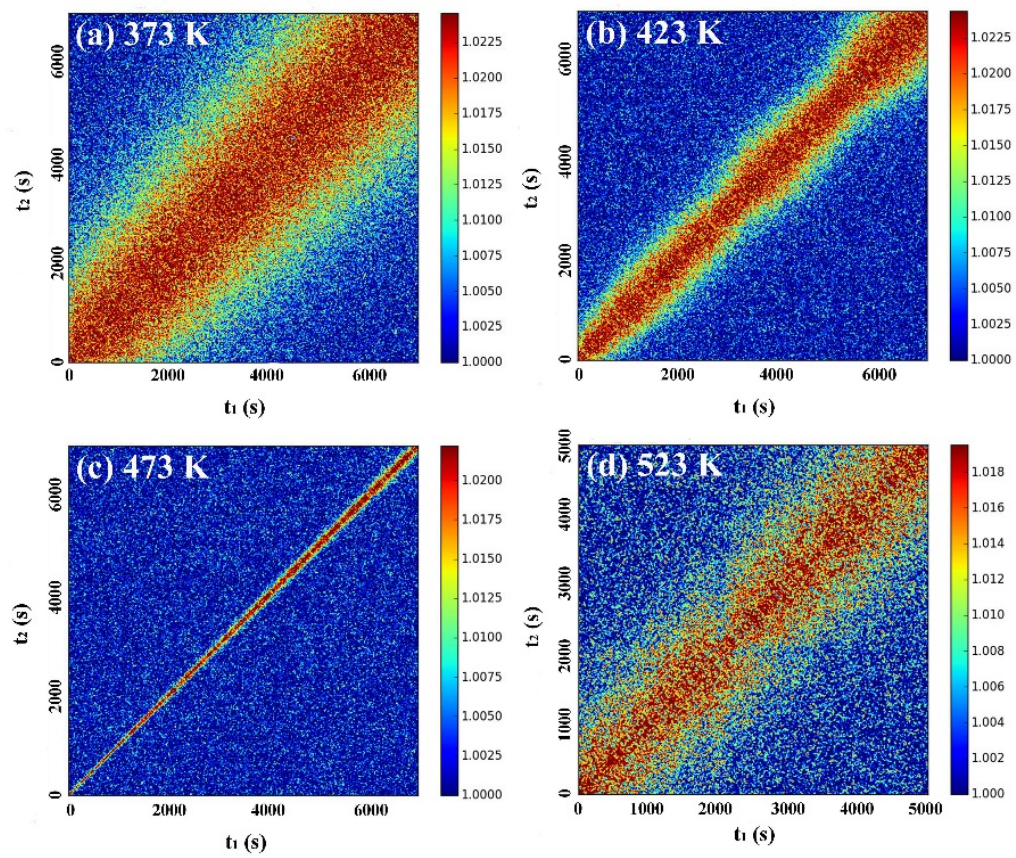
Fig. 1. Two-time correlation functions (TTCFs) for the annealed sample measured at a series of temperatures upon step heating: (a) slow and stationary dynamics at $373 \mathrm{~K}$, (b) accelerated and relatively stationary dynamics at 423K, (c) much faster dynamics with strong aging at $473 \mathrm{~K}$ and (d) decelerated stationary dynamics at $523 \mathrm{~K}$. The width of the diagonal profile is proportional to the relaxation time $\tau$.

The instantaneous dynamical scenario during the isothermal holding at each step temperature can be obtained by comparing the width change along the diagonal profiles in Fig. 1, and the dynamics at different step temperatures are also comparable. However, to evaluate the dynamics quantitatively, the normalized $\mathrm{g}_{2}(\mathrm{t})$ function as an average quantity of the dynamics over a progressively increasing waiting time was extracted, as shown in Fig. 2 (a). The $g_{2}(t)$ data are displayed together with the best fits to a model KWW function [5]. The values of the relaxation times are listed in Table 1 . Since the dynamics is not frozen in the glassy state, the normalized $\mathrm{g}_{2}(\mathrm{t})$ data displays a full decay to zero apart from the measurement at $290 \mathrm{~K}$ due to the extremely large relaxation time.
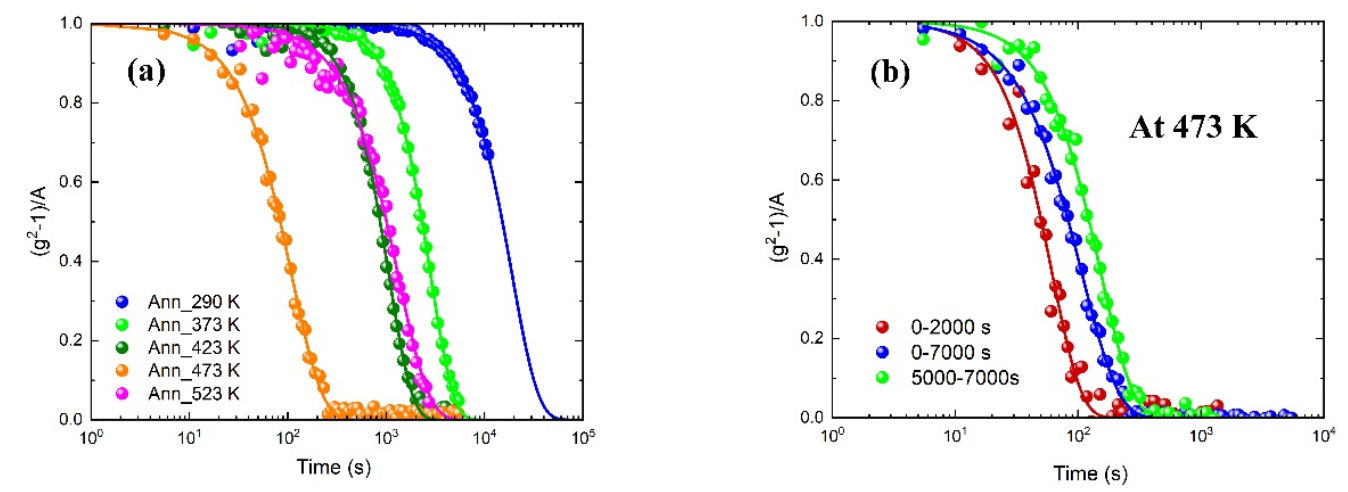

Fig. 2. (a) Normalized $g_{2}(t)$ (filled circles) of the annealed sample probed at different 
isothermal temperatures upon step heating from $290 \mathrm{~K}$, fitted by a model KWW function $f(t)=1+A \exp \left[-2(t / \tau)^{\beta}\right]$ (solid lines). Note, that the dynamics is basically the same at $423 \mathrm{~K}$ to $523 \mathrm{~K}$, and faster in between. Thus, this is denoted as dynamical “crossover”. (b) Normalized g2 (t) (filled circles) and the corresponding KWW fits (solid lines) of the annealed sample extracted from different stages during the isothermal process at $473 \mathrm{~K}$. The dynamics slows down and aging occurs.

\subsection{XPCS measurements on the HPT deformed state}

The aforementioned relaxed state (pre-annealing the as-cast state for 6 hours at $533 \mathrm{~K}$ ) was subjected to HPT deformation of 10 and 100 revolutions, and then the dynamics of the deformed samples were probed by XPCS at the same isothermal temperatures upon step heating from $290 \mathrm{~K}$. The normalized $\mathrm{g}_{2}(\mathrm{t})$ data, together with the best fits to a KWW model function, are shown in Fig. 3. The corresponding TTCFs and KWW exponents can be found in Fig. S2 and S3 in the Supplementary Materials. The fitting values of the relaxation times are listed in Table 1 as well. With respect to the HPT deformed sample, the dynamics is much faster and a full decay at each step temperature can be observed. 


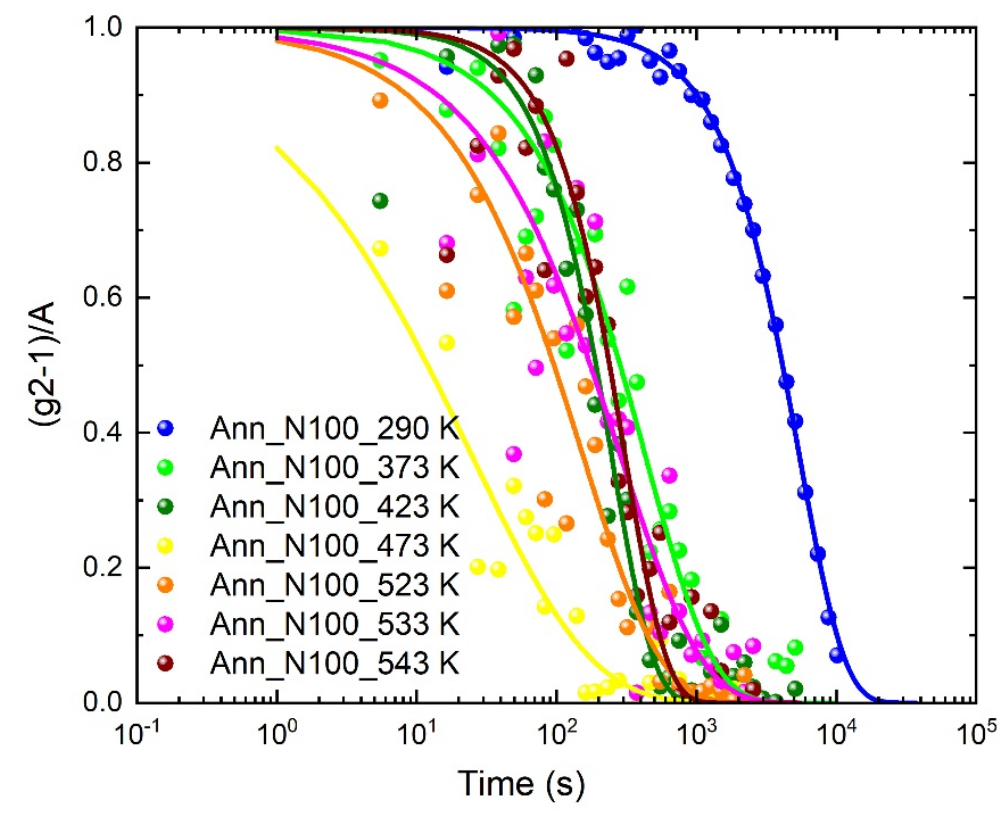

Fig. 3. Normalized $g_{2}(t)$ functions (filled circles) and the corresponding KWW fits (solid lines) of a HPT deformed sample (100 revolutions) probed at different isothermal temperatures upon step heating starting at $290 \mathrm{~K}$.

Fig. 4 shows the relaxation time $\tau$ of all the XPCS measurements as a function of temperature. Black and pink filled circles represent the relaxation times of the annealed and the Ann_N100 samples, respectively, and these two samples show a similar temperature-dependent dynamical trend. However, the decrease of the relaxation time by about a half order of magnitude at the same temperature indicates the occurrence of rejuvenation induced by HPT deformation. The filled triangles show the dynamics measured upon the subsequent slow cooling $(6 \mathrm{~K} / \mathrm{min})$ from the super-cooled regime. In addition, as shown by the green dashed line in Fig. 4, based on the structural relaxation parameters $\left(\tau_{0}=6.3 \times 10^{-14}, \mathrm{D}=5381\right.$ and $\left.\mathrm{T}_{0}=419\right)$ of $\mathrm{Pd}_{40} \mathrm{Ni}_{40} \mathrm{P}_{20} \mathrm{MG}$ in the 
super-cooled regime determined by dynamic mechanical analysis (DMA) and temperature modulated differential scanning calorimetry (TMDSC) measurements [44, 45], we modeled the temperature-dependent dynamics in the equilibrium state by fitting the data to a modified Vogel-Fulcher-Tammann (VFT) equation: $\tau(\mathrm{T})=\tau_{0} \exp \left[\frac{\mathrm{DT}_{0}}{\mathrm{~T}-\mathrm{T}_{0}}\right]$. Here $\mathrm{T}_{0}$ and $\mathrm{D}$ denote the temperature dependence of the liquid dynamics. A stronger liquid corresponding to a larger value of D more closely conforms to the Arrhenius law $[46,47]$. We can clearly observe the dynamical transition from the super-cooled regime into the out-of-equilibrium glassy state.

Table 1 . Values of the relaxation time $\tau$ obtained by fitting the $\mathrm{g}_{2}(\mathrm{t})$ data to a model KWW function.

\begin{tabular}{cccc}
\hline Measurements & $\tau(\mathrm{s})$ & Measurements & $\tau(\mathrm{s})$ \\
\hline Ann_290 K & 29103 & Ann_N100_473 K & 96 \\
Ann_373 K & 4086 & Ann_N100_523 K & 373 \\
Ann_423 K & 1554 & Ann_N100_533 K & 718 \\
Ann_473 K & 180 & Ann_N100_543 K & 496 \\
Ann_523 K & 2179 & Ann_N100_553 K & 278 \\
Ann_N10_290 K & 7621 & Ann_N100_563 K & 72 \\
Ann_N100_290 K & 9095 & Ann_N100_373 K_cooling & 4622 \\
Ann_N100_373 K & 929 & Ann_543 K_cooling & 1562 \\
Ann_N100_423 K & 400 & & \\
\hline
\end{tabular}




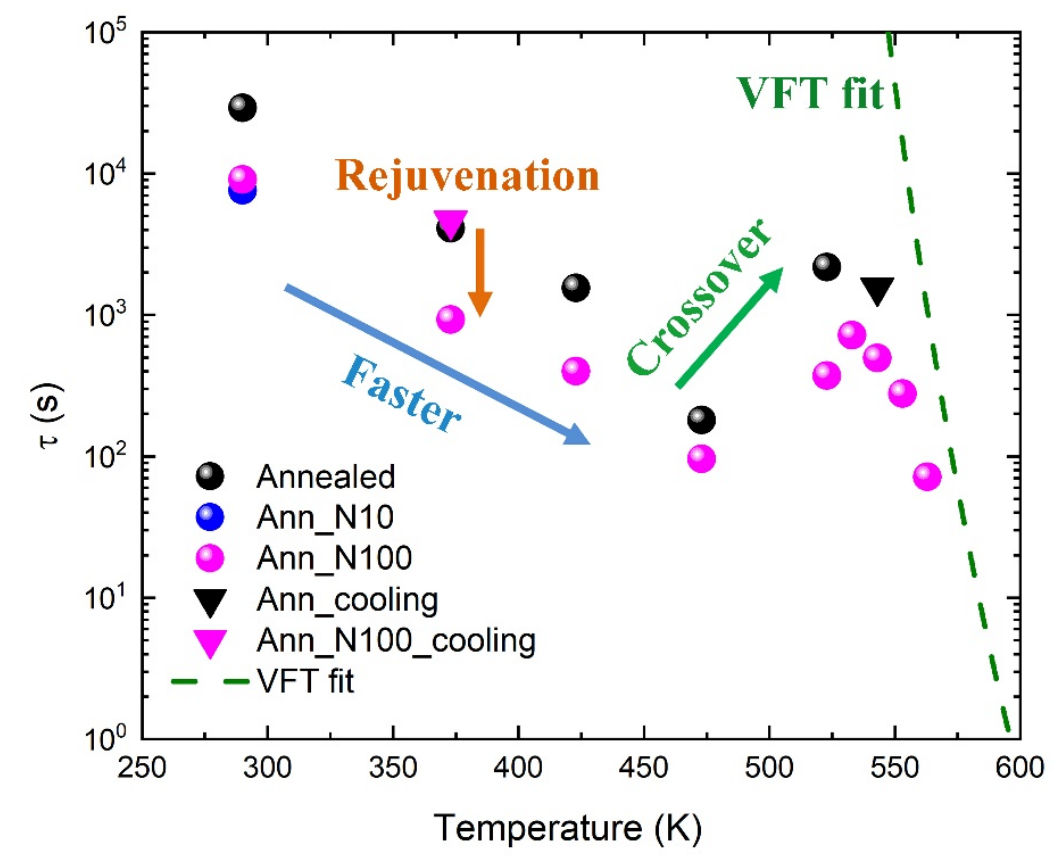

Fig. 4. Structural relaxation time $\tau$ obtained by XPCS measurements upon step heating from $290 \mathrm{~K}$ (filled circles) and subsequent cooling from the super-cooled regime (filled triangles) for the annealed sample (black color), the HPT sample deformed during 10 revolutions (blue color) and the HPT sample deformed during 100 revolutions (pink color). The green dashed line is a fit of the equilibrium data obtained by DMA and TMDSC measurements to the VFT equation [44, 45].

\subsection{Comparison of XPCS measurements with the calorimetric signals}

Fig. 5 shows the comparison of XPCS measurements at $290 \mathrm{~K}$ and DSC measurements of the annealed, N10 and N100 samples. From Fig. 5 (a), it is observed that the dynamics in Ann_N10 and Ann_N100 samples approximate each other, but are much faster than in the annealed system. With respect to the calorimetric properties, 
Ann_N10 and Ann_N100 samples show similar thermal reactions, and HPT deformation enhances the structural enthalpy (exothermal signal below $\mathrm{T}_{\mathrm{g}}$ ) and reduces the glass transition overshoot simultaneously.
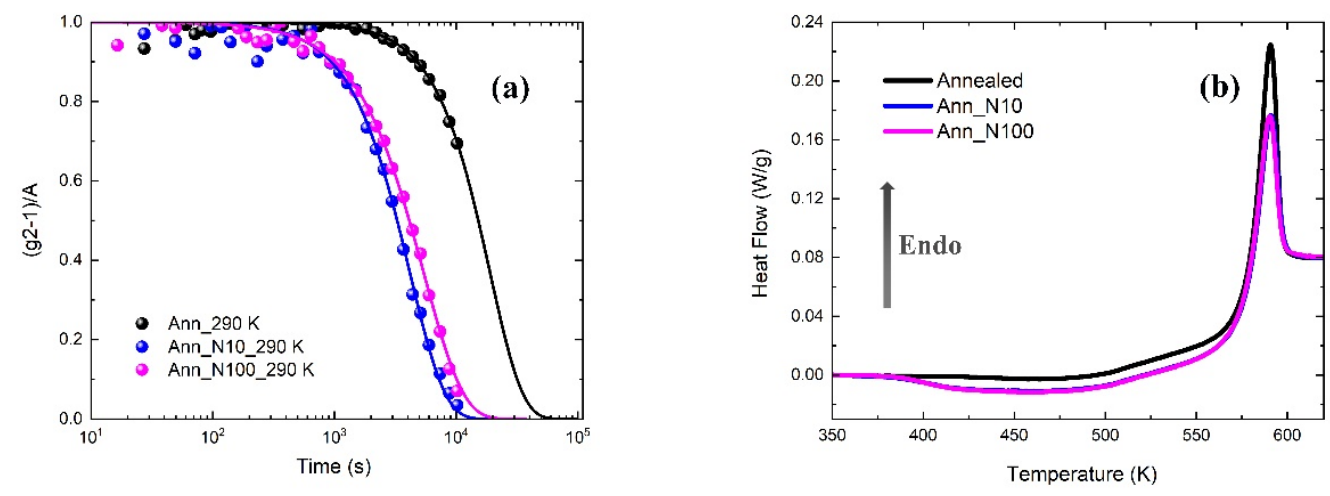

Fig. 5. (a) Normalized $g_{2}(t)$ for the annealed and the HPT deformed samples measured at $290 \mathrm{~K}$ and (b) DSC measurements (exotherm downwards) of the annealed and the HPT deformed samples of 10 revolutions and 100 revolutions.

3.4. VR-FEM measurements to characterize the MRO changes during HPT

It has been shown for a Zr-based BMG (Vit 105) that deformation alters the MRO significantly [27]. Here we attempt to analyze the MRO evolution in the Pd-based system prior and after severe plastic deformation achieved by HPT. Thus, the statistical information was extracted from individual NBDPs for the annealed, Ann_N10 and Ann_N750 foils, respectively. Normalized intensity variance profiles for each sample can be found in Fig. S4 (see Supplementary Materials). Here only the first peak maxima $\mathrm{V}$ were adopted and plotted as a function of $1 / \mathrm{R}^{2}$ as shown in Fig. 6, revealing two plateaus for the annealed sample (green color) and thus two MRO correlation lengths at 
about $1.3 \mathrm{~nm}$ and $3.2 \mathrm{~nm}$. Deformation by 10 revolutions (Ann_N10, orange color) shifts the level of the variance to higher values, which is due to a higher MRO volume fraction relative to the annealed sample without any deformation. However, one broad plateau, namely no apparent decrease or variation of the normalized variance is observed after 750 HPT revolutions (Ann_N750), which can be interpreted as a structurally homogeneous MG having a uniform distribution of MRO length scales. In order to observe the expected decrease of the normalized variance much larger probe sizes would be required.

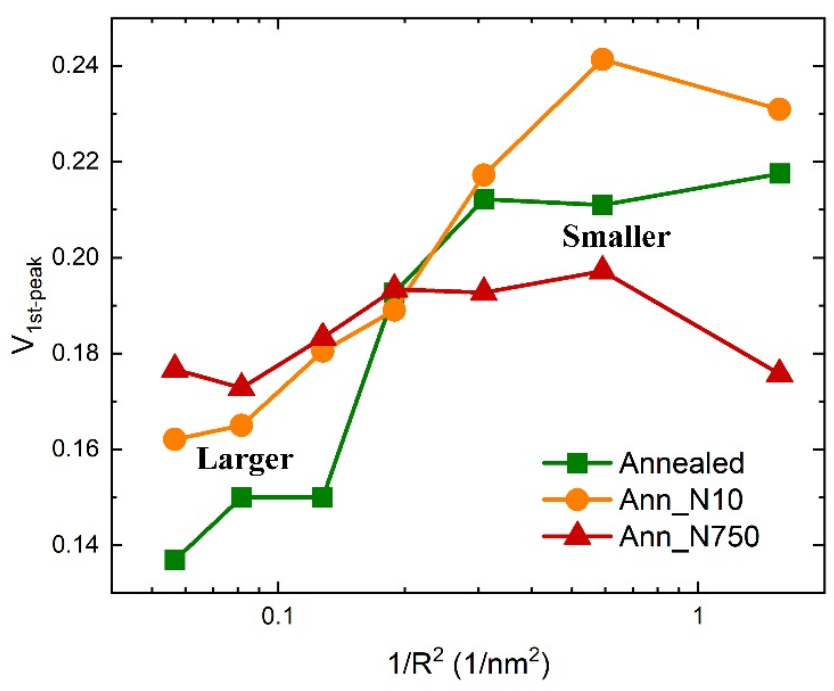

Fig. 6. MRO analysis according to the Stratton-Voyles approach [43] by plotting the first peak maxima $\mathrm{V}$ as a function of $1 / \mathrm{R}^{2}$. Note two plateaus revealing two correlation lengths for Ann_N10 and the annealed state. Extreme deformation after 750 HPT revolutions shows a homogeneous MRO length distribution over a wide range of probe sizes. 


\section{Discussion}

4.1. Variation of the structural relaxation dynamics

Due to the out-of-equilibrium state of MGs at temperatures sufficiently far below $T_{g}$, the local atomic structure slowly evolves towards a metastable equilibrium state with basically frozen mobility. This leads to structural relaxation and the annihilation of free volume [48-52]. Direct information on the relaxation dynamics related to the structural rearrangements was obtained by using the XPCS technique [16-18].

The time-resolved dynamics during holding at different temperature is shown in Fig. 1. Below $373 \mathrm{~K}$, aging is not apparent, and the relaxation dynamics is slow and approximately stationary within 7000 seconds of the experimental observation time. It is believed that the system with stationary dynamics is trapped in the deep energy basins of a potential energy landscape (PEL), and explores local minima separated by similar energy barriers $[14,18]$. Increasing the temperature to $423 \mathrm{~K}$ leads to an accelerated and stationary dynamical scenario. However, the slight fluctuation in the TTCF which manifests in a broadening and subsequent narrowing in the middle of the diagonal profile upon increasing the waiting time may resemble intermittent dynamics as found in other metallic glasses in a comparable temperature region [14]. This observation indicates the presence of heterogeneous dynamics due to the decorrelation of relaxation events contributed by multiple components. Further increase of the temperature to 473 $\mathrm{K}$ results in faster dynamics accompanied by strong aging, which is supposed to result 
from the gradual release of the atomic-level stresses by rearranging the localized atomic configurations. In this regime, the system's state can easily "escape" from a higher-energy basin, overcome the energy barrier and explore deeper energy minima. Besides, from Fig. 1 (b-d), it is observed that the TTCF contrast decreases as the temperature surpasses $473 \mathrm{~K}$, which happens simultaneously with the occurrence of fast relaxation dynamics and evident aging. This may be a consequence of the massive atomic rearrangements at the higher temperature, which change the local atomic configurations and thus decrease the diffracted intensity.

Quantitative information about the temperature-dependent and the deformation-influenced dynamics is obtained by analyzing the normalized $g_{2}(t)$ data in Figs. 2 and 3. The results are listed in Table 1 and plotted in Fig. 4. It is visible that the relaxation time of the glassy state with a basically frozen mobility displays a much weaker temperature dependence with respect to the ultra-viscous super-cooled liquid, whose properties can be well described by the empirical VFT equation, as indicated by the green dashed line in Fig. 4 [44]. In addition, the accelerated dynamics of HPT deformed samples is significant and it is termed "rejuvenation". This means that HPT deformation causes the MG system to be trapped in a higher energy basin of the PEL. In terms of the local atomic structure, this "rejuvenation" could be due to the introduction of additional excess free volume during deformation [53, 54]. However, the severe deformation processing could also disintegrate local structures that provided the 
conditions for “cage-like” slow dynamics. The rejuvenation effect can also be verified by the faster dynamics of the Ann_N100 sample measured at $373 \mathrm{~K}$ with respect to the subsequent measurement at the same temperature during slow cooling from the super-cooled liquid (the pink filled triangles).

In addition, Fig. 4 shows the accelerated dynamics with increasing temperatures. However, a remarkable dynamical “crossover” phenomenon occurs, reflected by the increasing values of $\tau$ from $473 \mathrm{~K}$ to $563 \mathrm{~K}$. To analyze this behavior, the activation energies in different temperature regimes were estimated by Arrhenius fits to the XPCS data, as shown in Fig. 7. At temperatures below $423 \mathrm{~K}$, the relaxation dynamics can be well described by an activation energy of $21.2 \mathrm{~kJ} / \mathrm{mol}$. Due to the low energy barrier for this process, it is believed that the relaxation at low temperatures mainly originates from the release of internal stress, namely the slight adjustments of atomic position without long-range atomic movements. HPT deformation can introduce localized stresses and free volume, which derive from the anisotropic arrangements of the atoms and may be released by reheating the system during a DSC scan $[48,51,55]$. The relaxation time at $473 \mathrm{~K}$ deviates from the Arrhenius fit because of the strong aging phenomenon, see Fig. 1 (c) and Fig. 2 (b), which makes the calculated $\tau$ at this temperature shorter than the extrapolation of the fit at temperatures below $423 \mathrm{~K}$ with the approximately stationary dynamics. It is supposed that aging is related to the anelastic internal stress. Above the "crossover" regime, the activation energy is $115.4 \mathrm{~kJ} / \mathrm{mol}$, estimated from the brown 
curve in Fig. 7. This is coincident with a typical activation energy of $\beta$ relaxation calculated from the empirical relationship $\mathrm{E}_{\beta}=26 \pm 2 \mathrm{RT}_{\mathrm{g}}$ [56]. $\beta$ relaxation usually manifests as a broad hump prior to the glass transition in Pd-based MGs. It is suggested to derive from the highly cooperative atomic motions of $10-20$ atoms in a string-like manner [56-58]. Moreover, the transition temperature of the relaxation mechanism corresponds to the start of the endothermal signal in Fig. 5 (b). The activation energy increases to $245.4 \mathrm{~kJ} / \mathrm{mol}$ upon further temperature increase. This value is in agreement with the energy barrier of the glass transition given in [59], suggesting extensive long-range atomic movements. Thus, it is concluded that the tremendous change of the energy barrier indicates a transition of the relaxation mechanism from a stress-dominated to a diffusion-dominated process with increasing temperature. This is in line with the results of creep experiments [60].

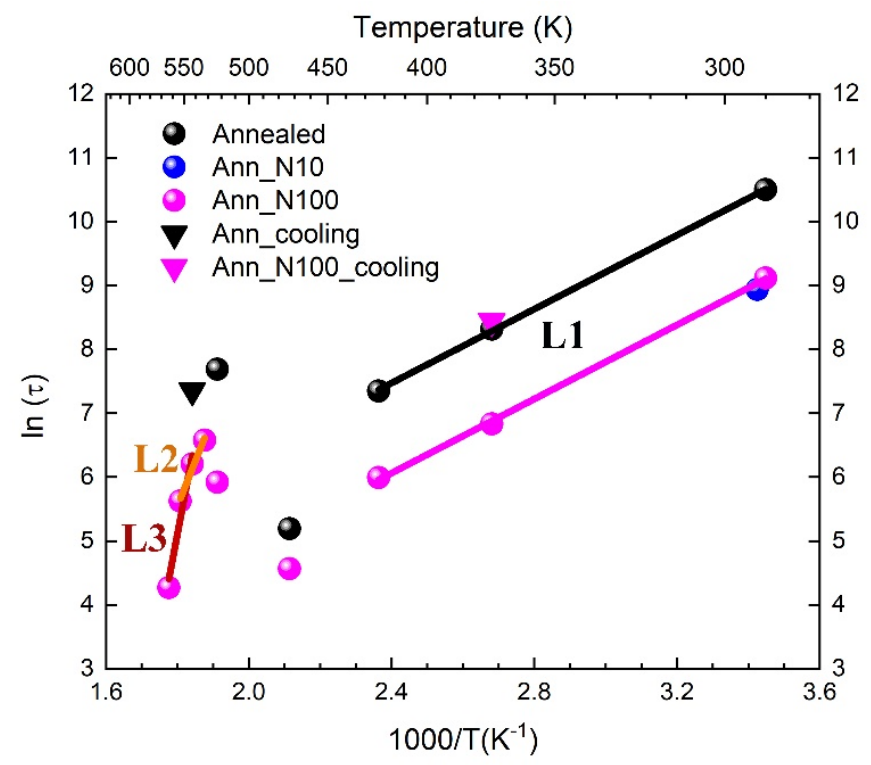

Fig. 7. Estimation of the activation energy. At temperatures below $423 \mathrm{~K}$ (black and 
pink lines 1), the activation energy amounts to approximately $21.2 \mathrm{~kJ} / \mathrm{mol}$. The estimated activation energy above $533 \mathrm{~K}$ (brown line 2) increases to $115.4 \mathrm{~kJ} / \mathrm{mol}$. Further increase in temperature leads to an estimated activation energy of $245.4 \mathrm{~kJ} / \mathrm{mol}$ (red line 3).

4.2. Atomic rearrangements during HPT and the structural relaxation below $\mathrm{T}_{\mathrm{g}}$ In monolithic MG systems, short-range order (SRO) and MRO play an essential role in the initiation and propagation of shear bands [27, 61, 62]. Sheng et al. proposed that MRO is formed by connecting and packing SROs in the form of units of fivefold symmetry, while the chemical SRO consists of solute-centered quasi-equivalent clusters. However, the quasi-equivalent clusters may display geometrical differences [63]. These geometrical differences can be resolved by motif extraction, which revealed that a collection of motifs coexist in the MG system and each motif possesses a certain population and a propensity of motion [64-67]. The glass transition or a plastic deformation process should be accompanied by a geometrical change of the motif configuration, resulting in an increase or decrease of the population of specific motifs, and should also be simultaneously accompanied by a change of the correlation length of motifs (i.e. the MRO size) [27]. During plastic deformation of MGs, shear transformation zones (STZs) are usually regarded as the deformation carriers [68, 69]. The activation process of STZ should lead to an increase of the population of motifs with higher propensity of motion, which can act as the main carrier of highly 
concentrated stress and subsequent heat release during stress relaxation. The proposed scenario that is based on a change of the distribution of motifs is supported by the VR-FEM results. Within the framework of the model proposed by Stratton and Voyles [43], the information on MRO clusters can be obtained by analyzing the change of the peak maximum of $\mathrm{V}$ as a function of $1 / \mathrm{R}^{2}$. Fig. 6 shows that there are two MRO correlation lengths present for the annealed (undeformed) sample which is in line with VR-FEM results obtained on PdNiP MGs [70, 71]. Subjected to a deformation by 10 revolutions of HPT, the plateau at the larger MRO size originally observed for the annealed state disappeared. It is notable that the sample deformed by 750 revolutions only displays one plateau with hardly any change in $\mathrm{V}$ for different probe sizes, which means that the configurations and MRO sizes are mostly uniformly distributed in this sample. Basically no probe size-dependence of the normalized variance has been observed and much larger probe sizes would be required to reveal the expected decrease towards the noise level. These topological changes characterized by the correlation length or the population of specific motifs result in the rejuvenation and accelerated dynamics induced by plastic deformation.

In this scenario, the stress-dominated relaxation mainly originates from the evolution of motifs from high to low propensity of motion by slightly adjusting the atomic position without detectable diffusion, which numerously occurs in the post-deformed glass $[64,65]$. Within this picture, the exothermic process that spans a wide temperature 
range as observed in the DSC measurements shown in Fig. 5 (b) corresponds to the gradual disappearance of stresses from a viscoelastic MG system. After annealing, due to the well-relaxed and homogenized state, numerous motifs with high propensity of motion were eliminated during annealing, and the XPCS results demonstrated that only a small fraction of atoms can be activated since the dynamics in the annealed system is slow. This result is in agreement with a small structural enthalpy released during the continuous heating below $\mathrm{T}_{\mathrm{g}}$ in Fig. 5 (b). For the HPT samples, when the population of motifs with high propensity of motion increases and rejuvenation occurs, relaxation dynamics can be accelerated dramatically. Numerous motifs with high propensity of motion in the vicinity of STZs can be activated having a high probability to evolve to the motifs with low propensity of motion, corresponding to the strongly enhanced exothermic enthalpy shown in Fig. 5 (b). It should be pointed out that apart from the stress-dominated relaxation contributing to the heat release, there exists diffusive relaxation observed by XPCS measurements contributing to the heat absorption below $\mathrm{T}_{\mathrm{g}}$, which is manifested as a broad endothermic hump below $\mathrm{T}_{\mathrm{g}}$ in Fig. 5 (b).

In summary, the relaxation scenario below $\mathrm{T}_{\mathrm{g}}$ for the annealed and HPT deformed samples is elucidated by XPCS measurements. Moreover, the current work illustrates the significance of the change of the topological structure during the deformation and relaxation of MGs.

\section{Conclusions}


In this work, combining XPCS, DSC and VR-FEM measurements, the dynamics of well relaxed (annealed) and severely HPT deformed $\mathrm{Pd}_{40} \mathrm{Ni}_{40} \mathrm{P}_{20}$ MGs was inspected on the atomic length scale at different isothermal temperatures. Furthermore, the connection between the measured relaxation dynamics and the associated structural rearrangements was explored. The results show that the relaxation dynamics of $\mathrm{Pd}_{40} \mathrm{Ni}_{40} \mathrm{P}_{20} \mathrm{MG}$ is accelerated by about a half order of magnitude through high-pressure torsion processing, which indicates the occurrence of strain-induced rejuvenation. The observed dynamical intermittence at $423 \mathrm{~K}$ indicates the presence of dynamical heterogeneity in this MG system. At temperatures below $473 \mathrm{~K}$, the activation energies of both undeformed and deformed samples are approximately $21.2 \mathrm{~kJ} / \mathrm{mol}$, which indicates that the relaxation process at low temperature is stress-dominated. Thus deformation increased the atomic mobility without changing the energy barrier. Above $473 \mathrm{~K}$, a "crossover" behavior of the effective relaxation time occurs, which is due to a transition of the relaxation mechanism from a stress-dominated to a diffusion-dominated process, accompanied by an activation energy increase to $245.4 \mathrm{~kJ} / \mathrm{mol}$. DSC and VR-FEM measurements revealed that the change of the topological structure (MRO and local motifs) plays a significant role during the deformation and relaxation of MGs.

\section{Acknowledgements}

This work was financially supported by German Research Foundation under the grant numbers: W1 1899/29-1, PE 2290/2-1 and INST 211/719-1 FUGG. We acknowledge F. 
Zontone (ESRF) for the technical support during the XPCS measurements and ESRF for providing beamtime at the beamline of ID10. Dr. Harald Rösner and PD Dr. Sergiy

Divinski are acknowledged for the kind discussions. H. Zhou is grateful to the China Scholarship Council for its financial support. E. Pineda acknowledges financial support from MINECO (grant FIS2017-82625-P) and Generalitat de Catalunya (grant 2017SGR0042).

\section{References}

[1] P. Murali, U. Ramamurty, Embrittlement of a bulk metallic glass due to sub-T-g annealing, Acta Mater 53(5) (2005) 1467-1478.

[2] M.E. Launey, R. Busch, J.J. Kruzic, Effects of free volume changes and residual stresses on the fatigue and fracture behavior of a Zr-Ti-Ni-Cu-Be bulk metallic glass, Acta Mater 56(3) (2008) 500-510.

[3] F. Alvarez, A. Alegria, J. Colmenero, Relationship between the Time-Domain Kohlrausch-Williams-Watts and Frequency-Domain Havriliak-Negami Relaxation Functions, Phys Rev B 44(14) (1991) 7306-7312.

[4] J.C. Qiao, J.M. Pelletier, Dynamic Mechanical Relaxation in Bulk Metallic Glasses: A Review, J Mater Sci Technol 30(6) (2014) 523-545.

[5] C.A. Angell, K.L. Ngai, G.B. McKenna, P.F. McMillan, S.W. Martin, Relaxation in glassforming liquids and amorphous solids, J Appl Phys 88(6) (2000) 3113-3157.

[6] R. Richert, Physical aging and heterogeneous dynamics, Phys Rev Lett 104(8) (2010) 085702.

[7] I. Binkowski, G. Shrivastav, J. Horbach, S. Divinski, G. Wilde, Shear band relaxation in a deformed bulk metallic glass, Acta Mater 109 (2016) 330-340.

[8] J.C. Mauro, S.S. Uzun, W. Bras, S. Sen, Nonmonotonic evolution of density fluctuations during glass relaxation, Phys Rev Lett 102(15) (2009) 155506.

[9] G. Afonin, Y.P. Mitrofanov, N. Kobelev, M. da Silva Pinto, G. Wilde, V. Khonik, Relationship between the enthalpies of structural relaxation, crystallization and melting in metallic glass-forming systems, Scripta Mater 166 (2019) 6-9.

[10] W. Jiang, F. Pinkerton, M. Atzmon, Mechanical behavior of shear bands and the effect of their relaxation in a rolled amorphous Al-based alloy, Acta materialia 53(12) (2005) 3469-3477.

[11] C. Nagel, K. Rätzke, E. Schmidtke, F. Faupel, W. Ulfert, Positron-annihilation studies of free-volume changes in the bulk metallic glass $\mathrm{Zr} 65 \mathrm{Al} 7.5 \mathrm{Ni} 10 \mathrm{Cu} 17.5$ during structural relaxation and at the glass transition, Physical review B 60(13) (1999) 9212.

[12] K. Hajlaoui, T. Benameur, G. Vaughan, A. Yavari, Thermal expansion and indentation-induced free volume in $\mathrm{Zr}$-based metallic glasses measured by real-time diffraction using synchrotron radiation, Scripta Materialia 51(9) (2004) 843-848. 
[13] Y. Ritter, K. Albe, Thermal annealing of shear bands in deformed metallic glasses: Recovery mechanisms in Cu64Zr36 studied by molecular dynamics simulations, Acta Materialia 59(18) (2011) 7082-7094.

[14] Z. Evenson, B. Ruta, S. Hechler, M. Stolpe, E. Pineda, I. Gallino, R. Busch, X-Ray Photon Correlation Spectroscopy Reveals Intermittent Aging Dynamics in a Metallic Glass, Phys Rev Lett 115(17) (2015) 175701.

[15] P. Heitjans, J. Kärger, Diffusion in condensed matter: methods, materials, models, Springer Science \& Business Media2006.

[16] M. Leitner, B. Sepiol, L.M. Stadler, B. Pfau, G. Vogl, Atomic diffusion studied with coherent X-rays, Nat Mater 8(9) (2009) 717-20.

[17] I. Gallino, D. Cangialosi, Z. Evenson, L. Schmitt, S. Hechler, M. Stolpe, B. Ruta, Hierarchical aging pathways and reversible fragile-to-strong transition upon annealing of a metallic glass former, Acta Mater 144 (2018) 400-410.

[18] B. Ruta, Y. Chushkin, G. Monaco, L. Cipelletti, E. Pineda, P. Bruna, V.M. Giordano, M. Gonzalez-Silveira, Atomic-scale relaxation dynamics and aging in a metallic glass probed by $x$-ray photon correlation spectroscopy, Phys Rev Lett 109(16) (2012) 165701.

[19] M. Luttich, V.M. Giordano, S. Le Floch, E. Pineda, F. Zontone, Y. Luo, K. Samwer, B. Ruta, Anti-Aging in Ultrastable Metallic Glasses, Phys Rev Lett 120(13) (2018) 135504.

[20] W. Dmowski, Y. Yokoyama, A. Chuang, Y. Ren, M. Umemoto, K. Tsuchiya, A. Inoue, T. Egami, Structural rejuvenation in a bulk metallic glass induced by severe plastic deformation, Acta Mater 58(2) (2010) 429-438.

[21] C.A. Schuh, T.C. Hufnagel, U. Ramamurty, Mechanical behavior of amorphous alloys, Acta Mater 55(12) (2007) 4067-4109.

[22] S. Pauly, S. Gorantla, G. Wang, U. Kühn, J. Eckert, Transformation-mediated ductility in CuZr-based bulk metallic glasses, Nat Mater 9(6) (2010) 473.

[23] H. Zhou, M. Peterlechner, S. Hilke, D. Shen, G. Wilde, Influence of plastic deformation by high-pressure torsion on the crystallization kinetics of a Pd40Ni40P20 bulk metallic glass, Journal of Alloys and Compounds (2019) 153254.

[24] E. Munch, J.-M. Pelletier, B. Sixou, G. Vigier, Characterization of the drastic increase in molecular mobility of a deformed amorphous polymer, Physical review letters 97(20) (2006) 207801.

[25] J.W. Deng, K. Du, M.L. Sui, Medium range order of bulk metallic glasses determined by variable resolution fluctuation electron microscopy, Micron 43(7) (2012) 827-31.

[26] S.N. Bogle, L.N. Nittala, R.D. Twesten, P.M. Voyles, J.R. Abelson, Size analysis of nanoscale order in amorphous materials by variable-resolution fluctuation electron microscopy, Ultramicroscopy $110(10)$ (2010) 1273-1278.

[27] S. Hilke, H. Rösner, D. Geissler, A. Gebert, M. Peterlechner, G. Wilde, The influence of deformation on the medium-range order of a $\mathrm{Zr}$-based bulk metallic glass characterized by variable resolution fluctuation electron microscopy, Acta Materialia 171 (2019) 275-281.

[28] M. Treacy, J. Gibson, L. Fan, D. Paterson, I. McNulty, Fluctuation microscopy: a probe of medium range order, Reports on Progress in Physics 68(12) (2005) 2899.

[29] J. Hwang, P. Voyles, Variable resolution fluctuation electron microscopy on Cu-Zr metallic glass using 
a wide range of coherent STEM probe size, Microscopy and Microanalysis 17(1) (2011) 67-74.

[30] N. Nollmann, I. Binkowski, V. Schmidt, H. Rösner, G. Wilde, Impact of micro-alloying on the plasticity of Pd-based bulk metallic glasses, Scripta Materialia 111 (2016) 119-122.

[31] G. Wilde, S. Klose, W. Soellner, G. Görler, K. Jeropoulos, R. Willnecker, H. Fecht, On the stability limits of the undercooled liquid state of Pd-Ni-P, Materials Science and Engineering: A 226 (1997) 434-438.

[32] G. Wilde, G. Görler, R. Willnecker, G. Dietz, Thermodynamic properties of Pd40Ni40P20 in the glassy, liquid, and crystalline states, Applied physics letters 65(4) (1994) 397-399.

[33] Y.P. Mitrofanov, M. Peterlechner, I. Binkowski, M.Y. Zadorozhnyy, I. Golovin, S. Divinski, G. Wilde, The impact of elastic and plastic strain on relaxation and crystallization of $\mathrm{Pd}-\mathrm{Ni}-\mathrm{P}$-based bulk metallic glasses, Acta Materialia 90 (2015) 318-329.

[34] H. Zhou, R. Hubek, M. Peterlechner, G. Wilde, Two-stage rejuvenation and the correlation between rejuvenation behavior and the boson heat capacity peak of a bulk metallic glass, Acta Materialia 179 (2019) 308-316.

[35] B. Kestel, Non-acid electrolyte thins many materials for TEM without causing hydride formation, Ultramicroscopy 19(2) (1986) 205-211.

[36] Y. Chushkin, C. Caronna, A. Madsen, A novel event correlation scheme for X-ray photon correlation spectroscopy, Journal of Applied Crystallography 45(4) (2012) 807-813.

[37] S. Kirsch, V. Frenz, W. Schartl, E. Bartsch, H. Sillescu, Multispeckle autocorrelation spectroscopy and its application to the investigation of ultraslow dynamical processes, J Chem Phys 104(4) (1996) 1758-1761.

[38] L. Cipelletti, D.A. Weitz, Ultralow-angle dynamic light scattering with a charge coupled device camera based multispeckle, multitau correlator, Rev Sci Instrum 70(8) (1999) 3214-3221.

[39] C.S. Kumar, X-ray and Neutron Techniques for Nanomaterials Characterization, Springer2016.

[40] P. Voyles, D. Muller, Fluctuation microscopy in the STEM, Ultramicroscopy 93(2) (2002) 147-159.

[41] M.M.J. Treacy, J.M. Gibson, L. Fan, D.J. Paterson, I. McNulty, Fluctuation microscopy: a probe of medium range order, Rep Prog Phys 68(12) (2005) 2899-2944.

[42] T.L. Daulton, K.S. Bondi, K.F. Kelton, Nanobeam diffraction fluctuation electron microscopy technique for structural characterization of disordered materials-Application to Al88- xY7Fe5Tix metallic glasses, Ultramicroscopy 110(10) (2010) 1279-1289.

[43] W. Stratton, P. Voyles, A phenomenological model of fluctuation electron microscopy for a nanocrystal/amorphous composite, Ultramicroscopy 108(8) (2008) 727-736.

[44] Z. Zhao, P. Wen, C. Shek, W. Wang, Measurements of slow $\beta$-relaxations in metallic glasses and supercooled liquids, Phys Rev B 75(17) (2007) 174201.

[45] G. Wilde, Direct measurement of the slow $\alpha$-relaxation modes of a metallic liquid near the glass transition, Applied Physics Letters 79(13) (2001) 1986-1988.

[46] C.A. Angell, Formation of glasses from liquids and biopolymers, Science 267(5206) (1995) 1924-1935.

[47] I.-R. Lu, G. Wilde, G. Görler, R. Willnecker, Thermodynamic properties of Pd-based glass-forming alloys, Journal of non-crystalline solids 250 (1999) 577-581.

[48] A. Taub, F. Spaepen, The kinetics of structural relaxation of a metallic glass, Acta Metallurgica 28(12)

(1980) 1781-1788. 
[49] Z. Evenson, R. Busch, Equilibrium viscosity, enthalpy recovery and free volume relaxation in a Zr44Ti11Ni10Cu10Be25 bulk metallic glass, Acta Mater 59(11) (2011) 4404-4415.

[50] E. Pineda, P. Bruna, B. Ruta, M. Gonzalez-Silveira, D. Crespo, Relaxation of rapidly quenched metallic glasses: effect of the relaxation state on the slow low temperature dynamics, Acta Mater 61(8) (2013) 3002-3011.

[51] A.R. Yavari, A. Le Moulec, A. Inoue, N. Nishiyama, N. Lupu, E. Matsubara, W.J. Botta, G. Vaughan, M. Di Michiel, Å. Kvick, Excess free volume in metallic glasses measured by X-ray diffraction, Acta Mater 53(6) (2005) 1611-1619.

[52] N. Zheng, G. Wang, L. Zhang, M. Calin, M. Stoica, G. Vaughan, N. Mattern, J. Eckert, In situ high-energy x-ray diffraction observation of structural evolution in a Ti-based bulk metallic glass upon heating, J Mater Res 25(12) (2010) 2271-2277.

[53] J. Qiao, J.-M. Pelletier, H. Kou, X. Zhou, Modification of atomic mobility in a Ti-based bulk metallic glass by plastic deformation or thermal annealing, Intermetallics 28 (2012) 128-137.

[54] J. Qiao, Q. Wang, J. Pelletier, H. Kato, R. Casalini, D. Crespo, E. Pineda, Y. Yao, Y. Yang, Structural heterogeneities and mechanical behavior of amorphous alloys, Progress in Materials Science 104 (2019) 250-329.

[55] R. Busch, E. Bakke, W. Johnson, Viscosity of the supercooled liquid and relaxation at the glass transition of the Zr46. 75Ti8. 25Cu7. 5Ni10Be27. 5 bulk metallic glass forming alloy, Acta Mater 46(13) (1998) 4725-4732.

[56] H.-B. Yu, K. Samwer, Y. Wu, W.H. Wang, Correlation between $\beta$ relaxation and self-diffusion of the smallest constituting atoms in metallic glasses, Phys Rev Lett 109(9) (2012) 095508.

[57] F. Faupel, W. Frank, M.-P. Macht, H. Mehrer, V. Naundorf, K. Rätzke, H.R. Schober, S.K. Sharma, H. Teichler, Diffusion in metallic glasses and supercooled melts, Rev Mod Phys 75(1) (2003) 237.

[58] J.D. Stevenson, P.G. Wolynes, A universal origin for secondary relaxations in supercooled liquids and structural glasses, Nat Phys 6(1) (2010) 62.

[59] T. Ichitsubo, E. Matsubara, S. Kai, M. Hirao, Ultrasound-induced crystallization around the glass transition temperature for Pd40Ni40P20 metallic glass, Acta materialia 52(2) (2004) 423-429.

[60] J. Qiao, Y.-J. Wang, L. Zhao, L. Dai, D. Crespo, J.-M. Pelletier, L.M. Keer, Y. Yao, Transition from stress-driven to thermally activated stress relaxation in metallic glasses, Physical Review B 94(10) (2016) 104203.

[61] A. Cao, Y. Cheng, E. Ma, Structural processes that initiate shear localization in metallic glass, Acta Mater 57(17) (2009) 5146-5155.

[62] Z. Zhu, L. Gu, G. Xie, W. Zhang, A. Inoue, H. Zhang, Z. Hu, Relation between icosahedral short-range ordering and plastic deformation in $\mathrm{Zr}-\mathrm{Nb}-\mathrm{Cu}-\mathrm{Ni}-\mathrm{Al}$ bulk metallic glasses, Acta Mater 59(7) (2011) 2814-2822.

[63] H. Sheng, W. Luo, F. Alamgir, J. Bai, E. Ma, Atomic packing and short-to-medium-range order in metallic glasses, Nature 439(7075) (2006) 419.

[64] M. Kumar, E. Nicholson, D.W. Kirk, S.J. Thorpe, C.V. Singh, Short-range structural origins of serration events in metallic glasses, Journal of Alloys and Compounds 787 (2019) 840-850.

[65] J. Ding, S. Patinet, M.L. Falk, Y. Cheng, E. Ma, Soft spots and their structural signature in a metallic glass, Proceedings of the National Academy of Sciences 111(39) (2014) 14052-14056. 
[66] J.J. Maldonis, A.D. Banadaki, S. Patala, P.M. Voyles, Short-range order structure motifs learned from an atomistic model of a Zr50Cu45Al5 metallic glass, Acta Materialia 175 (2019) 35-45.

[67] J.J. Maldonis, P.M. Voyles, Local Structure Controlling the Glass Transition in a Prototype Metal-Metalloid Glass, arXiv preprint arXiv:1901.07014 (2019).

[68] F. Spaepen, A microscopic mechanism for steady state inhomogeneous flow in metallic glasses, Acta metallurgica 25(4) (1977) 407-415.

[69] A. Argon, Plastic deformation in metallic glasses, Acta metallurgica 27(1) (1979) 47-58.

[70] F.A. Davani, S. Hilke, H. Rösner, D. Geissler, A. Gebert, G. Wilde, What renders bulk metallic glass ductile/brittle?--new insight from the medium-range order, arXiv preprint arXiv:2003.01368 (2020).

[71] A. Hassanpour, S. Hilke, S.V. Divinski, G. Wilde, Impact of Severe Deformation by HPT at Room Temperature on the Relaxation of the Glassy and the Supercooled Liquid States of PdNiP, Available at SSRN 3499071. 